\title{
Remodelado cardíaco en el modelo preclínico de falla cardíaca isquémica experimental por dislipidemia en el ratón SR-B1 KO/ApoER6 1h/h
}

\author{
Alejandra Lonis ${ }^{1}$, Jackeline Moya ${ }^{1}$, Jorge E Jalii ${ }^{1}$, Maria Paz Ocaranza ${ }^{1}$, \\ Attilio Rigotti ${ }^{2}$, Luigi Gabrielli. ${ }^{1}$ \\ División de Enfermedades Cardiovasculares1 y \\ Departamento de Nutrición y Diabetes2, Escuela de Medicina \\ P. Universidad Católica de Chile.
}

Financiamiento: Fondecyt 1161739

Introducción: Los ratones SR-B1 KO/ApoER6 1h/h que son alimentados con una dieta rica en grasas saturadas, desarrollan enfermedad coronaria aterosclerótica severa, complicaciones isquémicas e insuficiencia cardíaca, con alta mortalidad. Los estudios con este modelo se han enfocado fundamentalmente en la enfermedad coronaria y menos en el remodelado cardíaco. El objetivo del trabajo ha sido caracterizar el remodelado miocárdico, evaluar la evolución temporal de la función ventricular izquierda y la sobrevida asociada a enfermedad cardíaca por ateromatosis.

Método: Ratones homocigotos SR-B1 KO/ApoER6 $1 \mathrm{~h} / \mathrm{h}$ fueron alimentados por 8 semanas con dieta aterogénica o dieta normal y se comparó la sobrevida en ambos grupos. A las 4 semanas se realizó un ecocardiograma bidimensional. En los ratones eutanasiados se evaluó en la pared cardíaca fibrosis miocárdica y tamaño de los cardiomiocitos por morfometría, apoptosis con técnica de TUNEL e infiltración por células inflamatorias mononucleares (ED1) por inmunohistoquímica.

Resultados: En el grupo que recibió dieta aterogénica la sobrevida se redujo en 46,7\% ( $p<0.001$ ), debido a muerte súbita y a falla cardíaca progresiva. En este grupo, a las 4 semanas se observó dilatación de cavidades izquierdas y disminución de la fracción de eyección del ventrículo izquierdo en comparación con el grupo control $(79,3 \pm 1,3 \%$ vs $66 \pm 3,7 \%$, $\mathrm{p}<0,01)$. También se observó aumento de la masa cardíaca relativa de 2.1 veces $(\mathrm{p}<0,001)$ y del peso pulmonar relativo en $80 \%(\mathrm{p}<0,001)$, sin cambios en las dimensiones de los cardiomiocitos. En el miocardio de los ratones que recibieron dieta aterogénica hubo un aumento de la fibrosis cardíaca de 7.9 veces $(\mathrm{p}<0.01)$ y del número de cardiomiocitos apoptóticos en 55.9 veces $(\mathrm{p}<0.01)$, junto a un aumento del número de células inflamatorias mononucleares ED1.

Conclusiones: En el modelo de falla cardíaca severa de etiología isquémica con alta mortalidad en el ratón homocigoto SR-B1 KO/ApoER6 1h/h sometido a una dieta aterogénica, con falla cardíaca izquierda por disfunción sistólica, el remodelado patológico del miocardio está dado fundamentalmente por apoptosis y fibrosis. También se observa un aumento discreto de macrófagos en la pared cardíaca. Es posible que el edema parietal también pueda ser un mecanismo de remodelado relevante en este modelo.

Correspondencia:

Dr. Jorge E Jalil

jorgejalil1956@gmail.com, jjalil@med.puc.cl 


\section{Cardiac remodeling in experimental cardiac failure induced by hyperlipidemia in mice.}

SR-B1 KO/ApoER6 1h/h mice fed a high saturated fat diet develop severe coronary atheromatosis, and cardiac failure with a high mortality rate. Cardiac remodeling under these conditions has not been well studied.

Aim: To evaluate the time course of left ventricular function, cardiac remodeling and survival associated to the administration of an atherogenic diet.

Method: Homozygote SR-B1 KO/ApoER6 1h/h mice received an atherogenic diet for 8 weeks. Mice receiving a normal diet served as controls. Survival rate, myocardial fibrosis, cardiomyocyte size, apoptosis and infiltration by inflammatory or mononuclear cells were compared between groups. A TUNEL technique was used to evaluate apoptosis.

Results: A $46.7 \%$ survival reduction compared to controls was observed in the experimental group $(\mathrm{p}<0.01)$, due to left ventricular and atrial dilatation associated to a decrease in ejection fraction (79,3 \pm $1,3 \%$ vs $66 \pm 3,7 \%, \mathrm{p}<0,01$, respectively). Also, an increased cardiac weight, 2.6 times greater was observed in the experimental group, compared to controls. Mice receiving the atherogenic diet showed an $80 \%$ increased lung weight. There was no evident change in cardiomyocytes, but there was more (7.9 times) cardiac fibrosis $(\mathrm{p}<0.01)$ and 55.9 times more apoptotic cells. $(\mathrm{p}<0.01)$, along with a greater number of inflammatory cells and ED1 mononuclear cells

Conclusion: Mice receiving an atherogenic diet develop heart failure and reduced survival rate. This is associated with cardiac remodeling with underlying apoptosis an ventricular wall fibrosis. It is possible that wall edema might contribute to the observed cardiac remodeling. 


\section{Introducción:}

Un modelo preclínico de falla cardíaca por enfermedad coronaria utilizado en la evaluación de intervenciones que puedan impactar en el desarrollo de falla cardíaca, en sus mecanismos subyacentes y en la mortalidad cardiovascular es el modelo de ateroesclerosis experimental en ratones homocigotos que presentan una deficiencia en el receptor SR-BI de HDL y una baja expresión de un gen modificado de apolipoproteína $\mathrm{E}$ (ratones SR-B1 KO/ApoER6 1h/h o HypoE) y que, además, son alimentados con una dieta rica en grasas saturadas ${ }^{1-4}$. Este modelo se caracteriza por presentar altos niveles de colesterol plasmático, ateroesclerosis acelerada difusa, infartos al miocardio, falla cardíaca progresiva y alta mortalidad prematura por muerte súbita, cuando reciben dieta alta en grasas saturadas ${ }^{2-4}$.

El modelo se basa en el cruce de ratones knock out deficientes en el receptor SR-BI de HDL (SR-BI $\mathrm{KO})$ con ratones con apolipoproteína $\mathrm{E}$ hipomórfica (ApoeR61h/h), que expresan niveles reducidos de una isoforma apoE murina semejante a la isoforma apoE4 y presentan hipercolesterolemia inducida por la dieta ${ }^{4}$. Es un modelo de enfermedad coronaria aterosclerótica severa y de complicaciones isquémicas con alta morta$\operatorname{lidad}^{2,3}$, inducido por dieta aterogénica, lo que permite controlar el momento de inicio de la enfermedad, la duración, severidad y posiblemente su regresión ${ }^{4}$. En este modelo se ha observado aterosclerosis severa de la raíz aórtica con disminución del lumen, que puede atenuarse utilizando simultáneamente vitamina E y C como antioxidantes en la dieta, con mejoría de la sobrevida y en marcadores proinflamatorios ${ }^{2}$. En este modelo, la cardiopatía isquémica puede reducirse al disminuir la magnitud de la dieta aterogénica pero también puede acelerarse en condiciones de aislamiento social. ${ }^{5}$

Los reportes respecto a la evolución de la función ventricular izquierda y del remodelado miocárdico inicia$l^{4} s^{4}$, se caracterizaron por cardiomegalia significativa asociada a infartos múltiples del miocardio, fibrosis miocárdica, hipertrofia cardiomiocitaria, cambios en el segmento ST T del electrocardiograma (infra y supradesnivel), dilatación VI en el fin de sístole, aumento del grosor parietal y disminución de la fracción de acortamiento del $\mathrm{VI}^{4}$. Recientemente, se ha observado cardiomegalia con aumento del peso cardíaco aproximadamente 2 veces mayor que en los controles ${ }^{3}$. Sin embargo, no se ha efectuado en este modelo experimental un análisis más detallado y actualizado del remodelado miocárdico en relación con la función ventri- cular izquierda y con la mortalidad.

El objetivo del trabajo ha sido evaluar la evolución de la función ventricular izquierda y caracterizar el remodelado miocárdico en este modelo experimental relacionándolo con la sobrevida.

\section{Métodos:}

Animales y dieta. El protocolo y sus procedimientos fueron aprobados previamente por el Comité de Ética y Bienestar Animal institucionales. Se utilizaron inicialmente ratones heterocigotos SR-B1 KO/ApoER6 1h/h (proporcionados gentilmente por el Dr Attilio Rigotti, Departamento de Nutrición, Diabetes y Metabolismo, Pontificia Universidad Católica de Chile). Estos animales fueron cruzados con el fin de generar ratones homocigotos SRB1KO/apoER61h/h (machos y hembras), seleccionados por genotipificación. Los ratones se mantuvieron bajo condiciones controladas de luz, temperatura y humedad y recibieron agua y dieta normales (Chow diet, Prolab RMH3000; PMI feeds INC., St Louis, MO, USA). A las 12 semanas de vida los ratones fueron aleatorizados a uno de los 2 grupos experimentales: un grupo donde se mantuvo la dieta normal habitual (Grupo control) y un grupo que recibió, por 8 semanas, una dieta aterogénica $(1,25 \%$ colesterol, $15 \%$ grasas totales y $0,5 \%$ de ácido cólico; 57BB; Test Diet, St Louis, MO, USA) diluida al $60 \%$ en dieta normal para inducir enfermedad aterosclerótica ${ }^{2,3}$.

Sobrevida: Se observó la sobrevida de ambos grupos de ratones durante un período de hasta 8 semanas, después de iniciada la dieta aterogénica. Los animales se inspeccionaron diariamente evaluando el estado de salud mediante una pauta de supervisión diaria. El punto final humanitario se basó en un puntaje determinado en base al compromiso del bienestar animal usando como criterios el aspecto del pelaje, aspecto de la marcha espontánea, ingesta de agua y comida, del peso corporal, y actividad espontánea. Los animales que se observaron en mal estado y con puntajes elevados de acuerdo con la pauta de supervisión fueron eutanasiados. La sobrevida se determinó con el método de Kaplan-Meier Función ventricular izquierda: Se efectuó mediante ecocardiografía bidimensional sin sedación farmacológica, con lo que se reducen los posibles riesgos asociados a la sedación y los efectos de los fármacos en la función cardíaca. En forma previa los roedores fueron progresivamente acostumbrados al procedimiento a su 
ingreso al protocolo. Los ecocardiogramas se obtuvieron al momento de la aleatorización antes de iniciar la dieta normal o la dieta aterogénica, y después de 4 semanas con la dieta experimental (normal o aterogénica) utilizando un equipo Philips iE33 con un transductor de 2,5 MHz. Las determinaciones fueron realizadas en forma ciega de acuerdo a las recomendaciones de American Society of Echocardiography ${ }^{6-8}$. Se determinaron dimensiones de fin de sístole y fin de diástole VI, grosores de la pared VI, diámetro de la aurícula izquierda y de la aorta ascendente y se calcularon la fracción de acortamiento y la fracción de eyección del VI, esta última con fórmula de Simpson.

Obtención de tejido cardíaco. El corazón se extrajo rápidamente y se lavó en suero fisiológico. Se cortó un trozo transversal en la mitad del corazón para fijación en formalina e inclusión en Paraplast para cuantificación de hipertrofia cardiomiocitaria, de fibrosis y de apoptosis miocárdica. El miocardio restante se congeló en nitrógeno líquido para determinaciones posteriores. Evaluación de la hipertrofia cardíaca. El grado de hipertrofia cardíaca se evaluó por la masa cardíaca relativa en relación con el peso corporal final y también con relación a la longitud de la tibia.

Para la determinación de hipertrofia cardiomiocitaria se utilizaron cortes transversales de $5 \mu \mathrm{m}$ de grosor de ventrículo izquierdo (VI) previamente incluidos en paraplast y posteriormente teñidos con Hematoxilina-Eosina ${ }^{8,9}$. Los cortes se examinaron con microscopía de luz (Nikon eclipse E400) y las imágenes fueron obtenidas con una cámara Nikon DS Fi1 acoplada al microscopio. Se obtuvieron fotos de distintos campos aleatorios usando con aumento 40X, seleccionando los cardiomiocitos íntegros y mejor definidos transversalmente (100 células). Se midió y se registró el área (A, $\mu \mathrm{m} 2)$ y el perímetro $(\mathrm{P}, \mu \mathrm{m})$ de los cardiomiocitos, utilizando el programa Nis-Element ${ }^{8,9}$

Cuantificación de la fibrosis miocárdica. Cortes transversales de VI de $5 \mu \mathrm{m}$ de grosor fueron teñidos con picrosirio rojo ${ }^{8,9}$. Se adquirieron imágenes con una cámara digital acoplada a un microscopio. Con el objetivo 20X se tomaron, aproximadamente, 20 fotos y 30 fotos sucesivas en las zonas subendocárdica (S) y miocárdica $(\mathrm{M})$, respectivamente. Finalmente, se fijó el lente en una zona carente de tejido y se adquirió una foto con el propósito de identificar y eliminar espacios sin tejido dentro de la preparación. Las imá- genes fueron analizadas con un software diseñado en base al programa Matlab para medición de Colágeno miocárdico ${ }^{8,9}$. Finalmente, se determinó el promedio de la fracción volumétrica de colágeno total de todas las fotografías captadas por cada corazón (FVCT) y se expresó como como porcentaje.

Evaluación de la apoptosis cardíaca. El grado de apoptosis miocárdica se evaluó con la técnica de TUNEL (TdT-mediated -dUtp Nick End Labeling), que determina fragmentación del DNA y se basa en la presencia de cortes en el DNA que se identifican por medio de la terminal deoxinucleotidil transferasa (TdT), una enzima que cataliza la adición de dUTPs, los que son marcados secundariamente, identificándose así las células que han sufrido un daño severo en el DNA. Se utilizaron cortes transversales de $5 \mu \mathrm{m}$ de grosor de VI, fijados en formalina al $4 \%$ e incluidos en Paraplast, con posterior desparafinación, hidratación y tratamiento con EDTA $1 \mathrm{mM}$ - Tris $1 \mathrm{mM} \mathrm{pH} 8$ para la recuperación antigénica. El análisis con TUNEL se realizó con el kit de detección de muerte celular in situ, POD (Roche). La tinción se evaluó usando un microscopio óptico, donde las células con núcleos TUNEL positivos y con evidencia de marginación de la cromatina en la microscopía se consideraron apoptóticas ${ }^{10-12}$. Para calcular el porcentaje de células apoptóticas se contaron 400 células consecutivas en 20 campos secuenciales (40x), contando el número total de núcleos y el número de núcleos apoptóticos $^{12}$.

Detección de macrófagos/monocitos (células ED1). Se utilizó inmunotinción de cortes de miocardio de 5um de grosor que se deparafinaron. La Inmunotinción se realizó utilizando un anticuerpo monoclonal contra ED-1 (Biorad mouse anti-rat ED1 MCA 341R) y un kit comercial de inmunohistoquímica (ab64264, Abcam) siguiendo el protocolo señalado por el fabricante. Los cortes se contratiñeron con hematoxlina y se montaron con Entellan, un medio de montaje libre de agua. Para la cuantificación de células ED1 en el miocardio usamos una escala semicuantitativa de 1 a 5 que describimos previamente en roedores9. De acuerdo con la escala mencionada, 1 = no se observan células; $2=$ un nivel bajo de monocitos/macrófagos en el VI; 3 y $4=$ un número moderado y grande de monocitos/macrófagos en el VI y 5 = gran número de monocitos/macrófagos en el VI agrupados en sitios donde se observan cicatrices y áreas de fibrosis 9 . 
Análisis estadístico. Los datos obtenidos se expresaron como promedio \pm EEM. Los grupos experimentales estuvieron constituidos por 8 y 15 animales, grupo con dieta normal y grupo con dieta aterogénica, respectivamente. Las comparaciones se realizaron con prueba de $t$ de Student para muestras independientes. Un valor de $\mathrm{p}<0,05$ se consideró estadísticamente significativo.

\section{Resultados:}

Sobrevida. El seguimiento de ambos grupos experimentales fue por un período de 8 semanas desde el inicio de la dieta del protocolo (Grupo control, $\mathrm{n}$ inicial $=$ 8 y Grupo con dieta aterogénica, $\mathrm{n}$ inicial $=15$ ). En el grupo control ocurrió una muerte de manera accidental llegando todos los demás animales a completar 8 semanas de observación (sobrevida media 55,3 $\pm 0,8$ días). En el grupo de animales alimentados con dieta aterogénica, en cambio, se observó una alta mortalidad con sobrevida promedio de 29,5 $\pm 1,8$ días (disminución de la sobrevida media en 46,7\%, p < 0.001) (Figura 1). En el grupo que recibió dieta aterogénica 2 ratones fueron eutanasiados por deterioro muy importante del bienestar general por falla cardiaca. La mayoría de los animales alimentados con la dieta aterogénica murió por falla cardiaca progresiva muy rápidamente $(\mathrm{n}=5)$ o por muerte súbita $(\mathrm{n}=8)$.

Dimensiones y función ventricular izquierda: A las 4 semanas con la dieta experimental. Se evaluó la función
VI por ecocardiografía bidimensional. En los ratones que recibieron dieta aterogénica se observó un aumento significativo de los diámetros de fin de sístole y de fin de diástole del VI (en 17 y 13\%, respectivamente) y también del diámetro de la aurícula izquierda y una disminución muy importante de la función sistólica del VI, con caída de 13.3 y 11 unidades porcentuales en la fracción de eyección y de acortamiento del VI, respectivamente (Tabla 1). En este grupo experimental no se observaron modificaciones significativas de los grosores parietales del VI.

Tabla 1. Dimensiones cardíacas y función sistólica Vl a las 4 semanas con dieta normal o con dieta aterogénica

\begin{tabular}{|c|c|c|}
\hline & $\begin{array}{c}\text { Grupo control } \\
\text { con dieta } \\
\text { normal }(n=7)\end{array}$ & $\begin{array}{l}\text { Grupo con Dieta } \\
\text { aterogénica } \\
(\mathrm{n}=8)\end{array}$ \\
\hline Fracción de Eyección VI (\%) & $79,3 \pm 1,3$ & $66 \pm 3,7$ ** \\
\hline Fracción de Acortamiento VI (\%) & $42.0 \pm 1,2$ & $30,8 \pm 2,5$ *** \\
\hline Diámetro de fin de Sístole VI (mm) & $1,8 \pm 0,1$ & $2,5 \pm 0,2$ ** \\
\hline Diámetro de fin de Diástole VI (mm) & $3,2 \pm 0,1$ & $3,6 \pm 0,2$ ** \\
\hline Grosor Pared Septal del VI (mm) & $0,8 \pm 0,03$ & $0,8 \pm 0,04$ \\
\hline Grosor Pared Posterior del VI ( $\mathrm{mm}$ ) & $0,8 \pm 0,03$ & $0,8 \pm 0,03$ \\
\hline Diámetro aorta ascendente $(\mathrm{mm})$ & $1,53 \pm 0,07$ & $1,55 \pm 0,05$ \\
\hline Aurícula Izquierda (mm) & $1,98 \pm 0,06$ & $2,69 \pm 0,1 * \star *$ \\
\hline
\end{tabular}

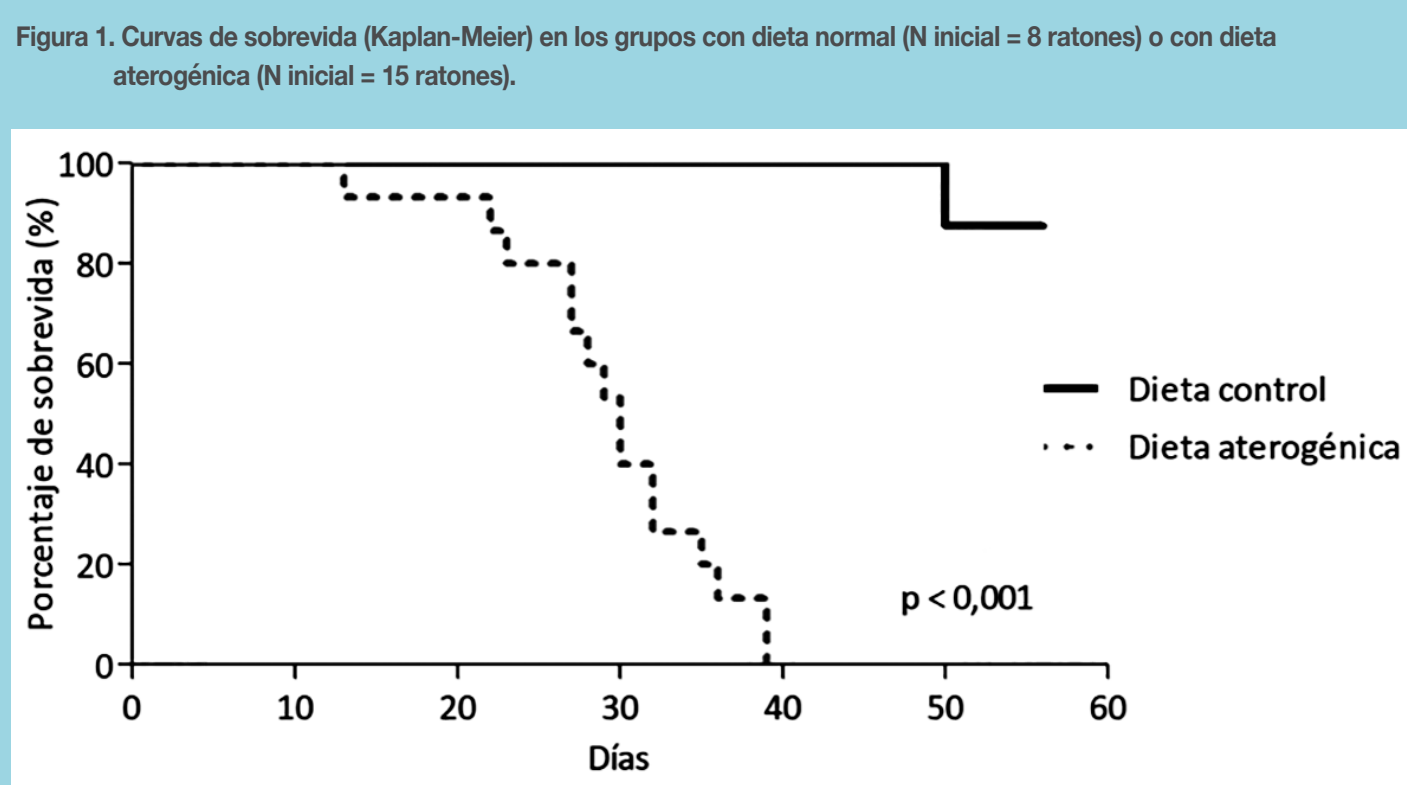


Tabla 2. Hipertrofia cardíaca y aumento de la masa pulmonar relativa en el grupo tratado con dieta aterogénica

\begin{tabular}{|c|c|c|}
\hline & $\begin{array}{l}\text { Grupo } \\
\text { control }\end{array}$ & $\begin{array}{c}\text { Grupo con Dieta } \\
\text { aterogénica }\end{array}$ \\
\hline Peso (gr) & $27,3 \pm 1,5$ & $22,3 \pm 0,7^{\text {** }}$ \\
\hline Masa cardíaca relativa/Peso & $0,53 \pm 0,03$ & $1,13 \pm 0,07$ *** \\
\hline Masa pulmonar relativa/Peso & $0,78 \pm 0,03$ & $1,46 \pm 0,08$ *** \\
\hline Longitud de la Tibia $(\mathrm{cm})$ & $1,8 \pm 0,02$ & $1,6 \pm 0,05$ ** \\
\hline Masa cardíaca relativa/Long Tibia & $8,24 \pm 0,81$ & $14,9 \pm 1,07^{\star \star \star}$ \\
\hline Masa pulmonar relativa/ Long Tibia & $12,2 \pm 0,81$ & $19,3 \pm 0,84$ *** \\
\hline
\end{tabular}

Masa cardíaca y masa pulmonar relativa. La Tabla 2 resume el peso, longitud de la tibia, la masa cardíaca relativa (MCR) y la masa pulmonar relativa de los ratones SRB1 KO/ApoER6 $1 \mathrm{~h} / \mathrm{h}$ alimentados con dieta normal y con dieta aterogénica. El peso de los ratones en dieta aterogénica fue significativamente menor en comparación a los ratones controles en $18 \%(\mathrm{p}<0,01)$, y la MCR fue 2.1 veces mayor en este grupo $(\mathrm{p}<0,001)$. La masa cardíaca relativa a la longitud de la tibia fue $80 \%$ mayor respecto del grupo control $(p<0,001)$. En el grupo de ratones que recibió dieta aterogénica se observó también aumento del peso pulmonar relativo de $58 \%$ ( $\mathrm{p}<0,001)$, muy posiblemente por aumento del agua extravascular pulmonar.

Fibrosis y apoptosis miocárdica. La fracción volumétrica de colágeno miocárdico (FVC) determinada por

Figura 2. Fibrosis y apoptosis miocárdica (en VI) en ratones alimentados con dieta control normal (DC) o con dieta aterogénica (DA)

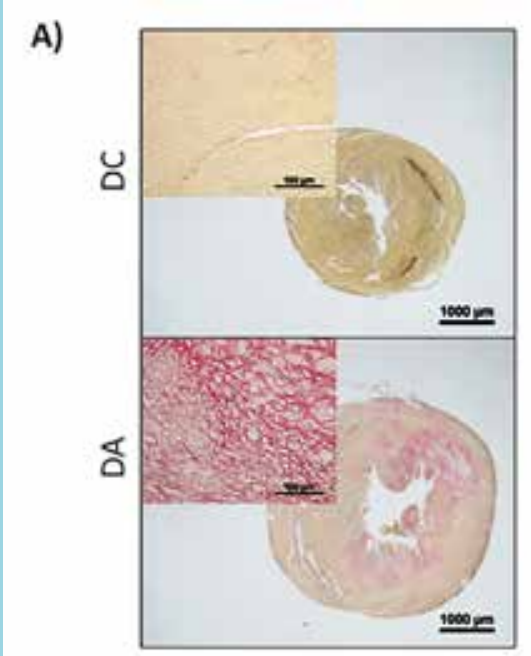

C)

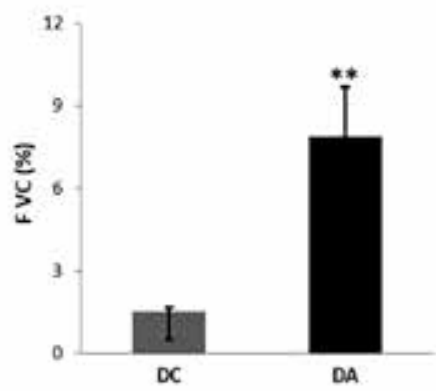

B)

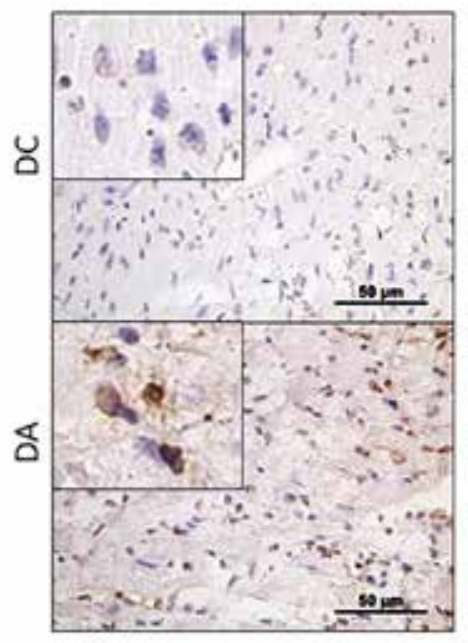

D)

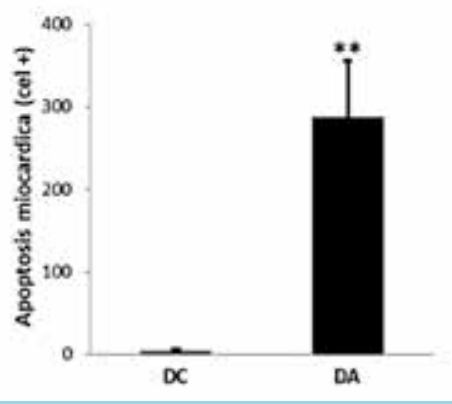

Fibrosis miocárdica con tinción de Picrosirio Rojo (imágenes representativas en A) y su cuantificación en ambos grupos como fracción volumétrica de colágeno $(F V C)$ miocárdico en gráfico inferior izquierdo en cortes trasversales de VI. Apoptosis (imágenes representativas de núcleos túnel-positivos en cardiomiocitos en ambos grupos, B) y Niveles de apoptosis miocárdica en ambos grupos experimentales gráfico inferior izquierdo. Resultados como promedio \pm ES. Símbolos $* *=p<0,01$. 
Figura 3. Aspecto del miocardio y de los cardiomiocitos con tinción de hematoxilina-eosina en los ratones alimentados con dieta control normal (DC) o con dieta aterogénica (DA).

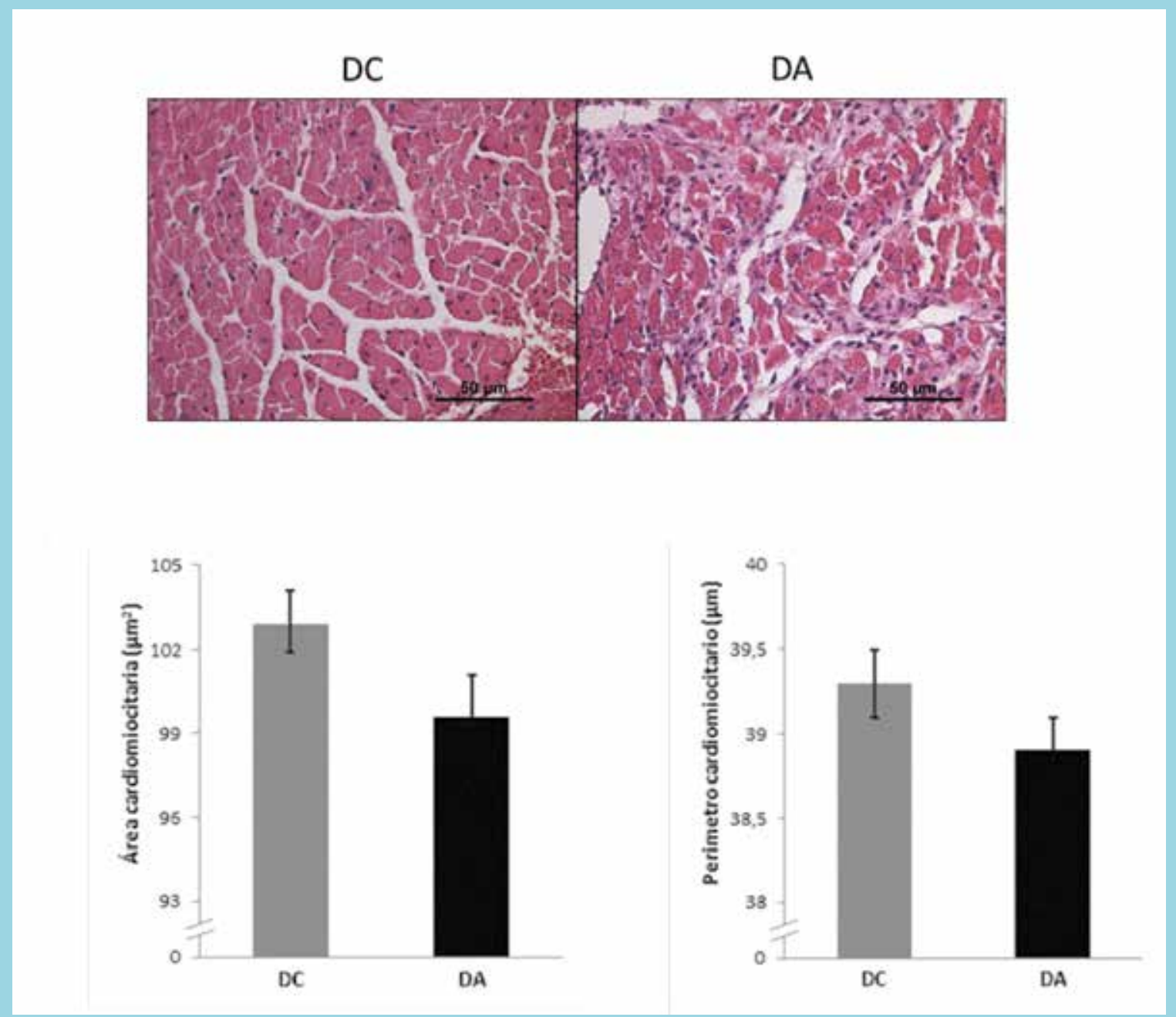

Gráficos del área (inferior izquierdo) y perímetro (inferior derecho) de los cardiomiocitos en ambos grupos. Resultados como promedio \pm ES.

morfometría fue significativamente mayor en los ratones que recibieron dieta aterogénica en comparación a los animales alimentados con dieta normal $(7,9 \pm 1,8$ y $1,5 \pm 0,18 \%$ respectivamente, $p<0.01$, (Figura $2 \mathrm{~A}$ ). El aumento del colágeno miocárdico se observó tanto en el subendocardio como en el miocardio.

Los ratones que recibieron la dieta aterogénica mostraron un marcado aumento en los niveles de apoptosis miocárdica determinada por TUNEL (Figura 2B) en comparación con los animales alimentados con dieta control $(287,7 \pm 67,5$ vs $5,14 \pm 1,58$ núcleos apoptóti$\cos \mathrm{p}<0.01$ (Figura 2B).

Área y perímetro de los cardiomiocitos. El aumento observado de la masa cardíaca relativa y de las dimen- siones del VI, no se acompañó de diferencias en el área ni en el perímetro de los cardiomiocitos en los ratones alimentados con la dieta aterogénica en comparación al grupo control (Figura 3).

Células mononucleares en el miocardio (macrófagos/ monocitos, ED1 positivas).

En ambos grupos experimentales se observó un número muy bajo de estas células inflamatorias en el miocardio, aunque 2.9 veces mayor en el grupo que recibió dieta aterogénica $(0.3 \pm 0.1$ vs $0.8 \pm 0.3, \mathrm{p}=0.053, \mathrm{n}=$ 7 y 6 animales por grupo).

\section{Discusión:}

Los hallazgos nuevos en el estudio son a nuestro juicio la marcada apoptosis miocárdica junto a fibrosis y au- 
mento leve de los macrófagos en la pared miocárdica. La activación o desencadenamiento de estos mecanismos celulares de remodelado cardíaco patológico, en este modelo de falla cardíaca de etiología isquémica, en el ratón homocigoto SRB1 KO/ApoER6 1h/h, se asoció a dilatación de cavidades cardíacas izquierdas y a disfunción sistólica del VI En este modelo experimental se desarrolla hipercolesterolemia cuando la alimentación es en base a una dieta aterogénica rica en grasas saturadas, con el subsecuente desarrollo de aterosclerosis coronaria severa ${ }^{2-4}$. Los fenómenos fisiopatológicos mencionados se asociaron a la alta mortalidad de causa cardíaca que observamos en el corto plazo en estos roedores.

Observamos ecocardiográficamente, a las 4 semanas con dieta aterogénica, un aumento importante de las dimensiones de fin de sístole y de diástole del VI, evidencia de deterioro contráctil. La consecuente sobrecarga de presión y volumen secundarias en este modelo de infartos múltiples del miocardio es diferente a lo observado en el modelo experimental de infarto secundario a ligadura de una arteria coronaria en roedores, donde la alteración parietal es segmentaria ${ }^{8}$. También se observó gran dilatación auricular izquierda, no cuantificada en estudios previos, secundaria muy probablemente a aumento de la presión de fin de diástole VI, aunque no realizamos mediciones hemodinámicas directas en el presente estudio.

La alta mortalidad en este modelo experimental está dada, en primer lugar, por falla cardíaca progresiva por disminución de la función sistólica con deterioro severo del bienestar animal. En los ratones que recibieron dieta aterogénica observamos aumento del peso de los pulmones por aumento del agua extravascular pulmonar propio de la falla cardíaca. La otra causa importante de mortalidad en este modelo es la muerte súbita, con toda seguridad por arritmias malignas. Leiva et al observaron muerte súbita en el $80 \%$ de los animales ${ }^{3}$. En nuestro medio, la sobrevida media observada en este modelo ha sido de 15 a 58 días $^{2,3}$ sin que se pueda establecer de manera totalmente precisa si la muerte en cada caso ocurre por arritmia maligna o por falla cardíaca progresiva rápida, para lo cual se requieren métodos de observación y monitoreo con otro nivel de precisión.

Las lesiones ateroscleróticas que se observan en este modelo preclínico han sido ampliamente descritas y caracterizadas. Corresponden a lesiones en la aorta, particularmente en los velos, en la pared de la aorta ascendente y en las arterias coronarias ${ }^{2-4}$, con lesiones miocárdicas ampliamente distribuidas en el surco AV, pared del VI, del VD y en el ápex ${ }^{4}$.

La aparición de fibrosis miocárdica ha sido descrita cualitativamente en este modelo, sin embargo no se ha sido cuantificada ${ }^{2-4}$. Utilizando morfometría observamos un aumento del colágeno miocárdico de, aproximadamente, 5 veces respecto de los controles alimentados con dieta normal, lo que señala de alguna manera la cronicidad de las lesiones. Desde el punto de vista fisiopatológico, consecuencias de la fibrosis miocárdica son la disfunción diastólica VI al reducirse la distensibilidad miocárdica ${ }^{13}$ y la disminución de la función contráctil $^{14}$ que hemos observado en estos ratones. Posiblemente, al aumentar patológicamente el colágeno miocardio el músculo cardíaco rodeado por colágeno se atrofia ${ }^{14}$, lo que lleva a disminución de la capacidad contráctil del miocardio, determinada en este modelo fundamentalmente por la pérdida de masa contráctil post IAM. Otra consecuencia muy importante de la fibrosis miocárdica en este modelo es la formación de áreas de heterogeneidad en el tejido cardíaco que favorecen el desarrollo de arritmias ventriculares malignas que pueden dar cuenta de por lo menos la mitad de las muertes observadas.

En los animales que recibieron la dieta aterogénica hubo aumento del peso cardíaco, tal como se ha reportado en diversos estudios en este modelo preclínico ${ }^{1-3}$. Sin embargo, ecocardiográficamente no observamos mayor grosor parietal en el VI y el área y diámetro de los cardiomiocitos fueron similares a los del grupo que recibió dieta. Es posible plantear que el aumento del peso cardíaco esté dado en parte significativa por edema de la pared del miocardio, lo que no cuantificamos en el presente estudio. En el modelo de isquemia-reperfusión en el ratón, donde se produce también necrosis miocárdica y disminución de la función sistólica VI, se ha observado edema de la pared miocárdica utilizando resonancia nuclear magnética cardíaca y cuantificado el edema en base a aumento de la secuencia T2, que se correlaciona con la función sistólica $\mathrm{VI}^{15}$. En el modelo experimental de isquemia-reperfusión en ratones se ha reportado que el edema miocárdico comprende aproximadamente el $50 \%$ de la masa ventricular izquierda en forma inicial, que va disminuyendo en el curso de los días a medida que se va produciendo la cicatrización, para comprometer posteriormente cerca del $25 \%$ de la misma ${ }^{15}$.

La apoptosis es un mecanismo de muerte celular con 
integridad de la membrana. En pacientes con IAM el grado de apoptosis miocárdica se correlaciona con oclusión persistente de la arteria coronaria culpable, remodelado VI desfavorable, e insuficiencia cardíaca precoz post IAM $^{16,17}$. En el infarto experimental en ratón por ligadura de la arteria descendente anterior se ha observado aumento de la expresión de la proteína proapoptótica BAX1 junto a un aumento de células TUNEL positivas en el área infartada, lo que podría estar mediado por activación de Rho kinasa y también por la vía dependiente del micro RNA mir200a ${ }^{18}$. De manera semejante, en el modelo de isquemia-reperfusión en el ratón se ha observado aumento del número de cardiomiocitos apoptóticos (TUNEL positivos) y aumento de los niveles de caspasa 3 clivada (Western blot) en el miocardio ${ }^{19}$. Sin embargo, no se ha descrito apoptosis miocárdica en el modelo de cardiopatía isquémica en ratones homocigotos SRB1KO/apoER$61 \mathrm{~h} / \mathrm{h}$ alimentados con dieta aterogénica. Dada la magnitud de la diferencia observada en nuestro estudio, el aumento muy marcado (de aproximadamente 56 veces) del número de cardiomiocitos apoptóticos en relación con el grupo control, planteamos que la apoptosis está jugando un rol importante en el deterioro observado de la función ventricular izquierda, en la falla cardíaca y en la mortalidad. Posiblemente, y aunque no lo determinamos en el estudio, también esté ocurriendo paralelamente necrosis miocárdica como otro mecanismo de pérdida de cardiomiocitos y deterioro funcional cardíaco en este modelo.

En este modelo experimental de falla cardíaca de etiología isquémica originalmente se describió "infiltración de células inflamatorias" en cortes de miocardio teñidos con tricromo Masson, sin hacer una aproximación cuantitativa ni tampoco más específica con respecto al tipo de células inflamatorias observadas ${ }^{4}$. En estudios más recientes utilizando este modelo experimental esto tampoco se ha analizado ${ }^{1-3}$. En los ratones que recibieron dieta aterogénica y que desarrollaron falla cardíaca isquémica observamos un número sobre 2 veces mayor (aunque bajo) de células ED 1 (células mononucleares que corresponden fundamentalmente a macrófagos), con respecto al grupo control. Su significado biológico es difícil de interpretar pero sin duda, por los otros procesos de remodelamiento patológico observados, hay en este modelo un rol patogénico adicional atribuible a la infiltración mononuclear. El estudio fue diseñado con un cálculo muestral para comparar sobrevida y el poder estadístico de la comparación del número de células ED 1 en el miocardio es bajo (51.7\%). En este mismo modelo se ha observado expresión de los genes proinflamatorios IL-6 y NF-kB que se reduce al administrar el anticoagulante oral rivaroxabán, pero sin establecerse comparaciones respecto de un grupo control ni determinarse los niveles de las proteínas respectivas ${ }^{1}$.

En resumen, en el modelo de falla cardíaca severa de etiología isquémica con alta mortalidad en el ratón homocigoto SRB1 KO/ApoER6 1h/h sometido a una dieta aterogénica, con falla cardíaca izquierda por disfunción sistólica, el remodelado patológico del miocardio se debe fundamentalmente a apoptosis y a fibrosis y, también, a un aumento discreto de macrófagos en la pared cardíaca. La presencia de edema parietal también puede ser un mecanismo de remodelado relevante en este modelo. Posibles vías de señalización que participen en estos procesos son la activación de Rho kinasa y la vía dependiente del micro RNA mir200a, que se han planteado como blancos terapéuticos.

\section{Referencias}

1. LIU J, NISHIDA M, INUI H, CHANG J, ZHU Y, KANNO K, MATSUDA H, SAIRYO M, OKADA T, NAKAOKA H, OHAMA T, MASUDA D, KOSEKI M, YAMASHITA S, SAKATA Y. Rivaroxaban Suppresses the Progression of Ischemic Car- diomyopathy in a Murine Model of Diet-Induced Myocardial Infarction. J Atheroscler Thromb. 2019;26:915-930.

2. CONTRERAS-DUARTE $S$, CHEN P, ANDÍA M, URIBE S, 
IRARRÁZAVAL P, KOPP S, KERN S, MARSCHE G, BUSSO D, WADSACK C, RIGOTTI A. Attenuation of atherogenic apo B-48-dependent hyperlipidemia and high density lipoprotein remodeling induced by vitamin $\mathrm{C}$ and $\mathrm{E}$ combination and their beneficial effect on lethal ischemic heart disease in mice. Biol Res;51(1):34. doi: 10.1186/s40659-018-0183-6.

3. LEIVA A, CONTRERAS-DUARTE S, AMIGO L, SEPÚLVEDA E, BORIC M, QUIÑONES V, BUSSO D, RIGOTTI A. Gugulipid causes hypercholesterolemia leading to endothelial dysfunction, increased atherosclerosis, and premature death by ischemic heart disease in male mice. PLoS One. 2017 Sep 14;12(9):e0184280. doi: 10.1371/journal.pone.0184280.

4. ZHANG S, PICARD MH, VASILE E, ZHU Y, RAFFAI RL, WEISGRABER KH, et al. Diet-induced occlusive coronary atherosclerosis, myocardial infarction, cardiac dysfunction, and premature death in scavenger receptor class B type I-deficient, hypomorphic apolipoprotein ER61 mice. Circulation. 2005;111:3457-3464.

5. NAKAGAWA-TOYAMA Y, ZHANG S, KRIEGER M. Dietary manipulation and social isolation alter disease progression in a murine model of coronary heart disease. PLoS One. 2012;7(10):e47965. doi: 10.1371/journal.pone.0047965.

6. LANG RM, BIERIG M, DEVEREUX RB, et al. Recommendations for chamber quantification: a report from the American Society of Echocardiography's Guidelines and Standards Committee and the Chamber Quantification Writing Group, developed in conjunction with the European Association of Echocardiography, a branch of the European Society of Cardiology. J Am Soc Echocardiogr. 2005;18:1440-1463.

7. VARGAS JF, GABRIELLI L, MANCILLA C, FERNÁNDEZ R, OLIVARES G, MOLINA M, MOYA J, VERDEJO H, OCARANZA MP, JALIL JE. En el modelo preclínico de insuficiencia cardíaca por disfunción sistólica en ratón (coartación de aorta torácica) la velocidad de flujo carotídeo determinada precozmente se asocia a función ventricular izquierda tardía. Rev Chil Cardiol 2018; 37: 194-200

8. MERA C, GODOY I, RAMÍREZ R, MOYA J, OCARANZA MP, JALIL JE. Mechanisms of favorable effects of Rho kinase inhibition on myocardial remodeling and systolic function after experimental myocardial infarction in the rat. Ther Adv Cardiovasc Dis. 2016;10:4-20.

9. OCARANZA MP, FIERRO C, JALIL JE, MOYA J, GONZALEZ L, MOLINA C, MANCILLA C. Rho kinase activation in circulating leukocytes is related to hypertensive myocardial remodeling. Clin Sci (Lond). 2018;132:1837-1853.

10. SLEE EA, ADRAIN C, MARTIN SJ. Executioner caspase-3, -6, and -7 perform distinct, non-redundant roles during the demolition phase of apoptosis. J Biol Chem. 2001;276:7320-7326.

11. Zhao Y, Jiang ZF, Sun YL, Zhai ZH. Apoptosis of mouse liver nuclei induced in the cytosol of carrot cells. FEBS Lett. 1999;448:197-200

12. OCARANZA MP, MOYA J, JALIL JE, LAVANDERO S, KALERGIS AM, MOLINA C, GABRIELLI L, GODOY I, CÓRDOVA S, CASTRO P, MAC NAB P, ROSSEL V, GARCÍA L, GONZÁLEZ J, MANCILLA C, FIERRO C, FARÍAS L. Rho-kinase pathway activation and apoptosis in circulating leucocytes in patients with heart failure with reduced ejection fraction. J Cell Mol Med. 2020;24:1413-1427.

13. JALIL JE, DOERING CW, JANICKI JS, PICK R, SHROFF SG, WEBER KT. Fibrillar collagen and myocardial stiffness in the intact hypertrophied rat left ventricle. Circ Res. 1989;64:1041-50.

14. JALIL JE, JANICKI JS, PICK R, ABRAHAMS C, WEBER KT. Fibrosis-induced reduction of endomyocardium in the rat after isoproterenol treatment. Circ Res. 1989;65:258-64.

15. BÖNNER F, JACOBY C, TEMME S, BORG N, DING Z, SCHRADER J, FLÖGEL U. Multifunctional MR monitoring of the healing process after myocardial infarction. Basic Res Cardiol. 2014;109(5):430. doi: 10.1007/s00395-014-0430-0.

16. ABBATE A, BUSSANI R, BIONDI-ZOCCAI GG, ROSSIELLO R, SILVESTRI F, BALDI F, BIASUCCI LM, BALDI A. Persistent infarct-related artery occlusion is associated with an increased myocardial apoptosis at postmortem examination in humans late after an acute myocardial infarction. Circulation. 2002;106:1051-4.

17. ABBATE A, BIONDI-ZOCCAI GG, BUSSANI R, DOBRINA A, CAMILOT D, FEROCE F, ROSSIELLO R, BALDI F, SILVESTRI F, BIASUCCI LM, BALDI A. Increased myocardial apoptosis in patients with unfavorable left ventricular remodeling and early symptomatic post-infarction heart failure. J Am Coll Cardiol. 2003;41:753-60.

18. WANG Y, JIANG Y, SUN X, SHEN X, WANG H, DONG C, LU B, YAN Y, LU Y, FASAE MB, LIU B, BAI Y. Downregulation of miR-200a protects cardiomyocyte against apoptosis. Biomed Pharmacother. 2020 Mar;123:109303. doi:10.1016/j. biopha.2019.109303.

19. ZHOU X, XIA N, LV B, TANG T, NIE S, ZHANG M, JIAO J, LIU J, XU C, HOU G, YANG X, HU Y, LIAO Y, CHENG $\mathrm{X}$. Interleukin 35 ameliorates myocardial ischemia-reperfusion injury by activating the gp130-STAT3 axis. FASEB J. 2020;34:3224-3238. 